\title{
Aligning nutrient profiling with dietary guidelines: modifying the Nutri-Score algorithm to include whole grains
}

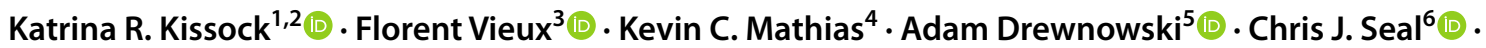

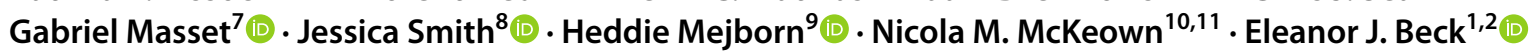

Received: 29 March 2021 / Accepted: 18 October 2021 / Published online: 24 November 2021

(c) The Author(s) 2021, corrected publication 2021

\begin{abstract}
Purpose Whole grains, generally recognised as healthy choices, are not included in most nutrient profiling systems. We tested modifications to the Nutri-Score algorithm to determine whether including whole grains would provide an improved measure of food, and overall diet quality.

Methods The whole-grain content of food, with a minimum cut-point of $25 \%$, was added to the algorithm, following similar methods used to score other health-promoting components such as fibre. We applied and compared the original and the modified Nutri-Score to food composition and dietary intake data from Australia, France, the United Kingdom, and the United States.

Results At the food level, correlations between whole-grain content and food nutritional score were strengthened using the modified algorithm in Australian data, but less so for the other countries. Improvements were greater in grain-specific food groups. The largest shift in Nutri-Score class was from B to A (best score). At the dietary intake level, whole-diet nutritional scores for individuals were calculated and compared against population-specific diet-quality scores. With modifications, correlations with diet-quality scores were improved slightly, suggesting that the modified score better aligns with national dietary guidelines. An inverse linear relationship between whole-diet nutritional score and whole-grain intake was evident, particularly with modifications (lower whole-diet nutritional score indicative of better diet quality).

Conclusion Including a whole-grain component in the Nutri-Score algorithm is justified to align with dietary guidelines and better reflect whole grain as a contributor to improved dietary quality. Further research is required to test alternative algorithms and potentially other nutrient profiling systems.
\end{abstract}

Keywords Whole grain $\cdot$ Intake $\cdot$ Nutrient profiling $\cdot$ Nutri-Score $\cdot$ Nutrient density

\section{Abbreviations}

AUSNUT Australian Food, Supplement and Nutrient Database

DALY Disability adjusted life years
FAQ Frequently asked questions

FNDDS Food and nutrient database for dietary studies FOP Front-of-pack

FPED Food Patterns Equivalent Database
Eleanor J. Beck

eleanor@uow.edu.au

1 School of Medicine, Faculty of Science, Medicine and Health, University of Wollongong, Wollongong, NSW 2522, Australia

2 Illawarra Health and Medical Research Institute, Wollongong, NSW, Australia

3 MS-Nutrition, Marseille, France

4 Skidmore College, Health and Human Physiological Sciences, Saratoga Springs, NY, USA

5 Center for Public Health Nutrition, University of Washington, Seattle, WA, USA
6 Public Health Sciences Institute, University of Newcastle, Newcastle upon Tyne NE2 4HH, UK

7 Cereal Partners Worldwide, Prilly, Switzerland

8 General Mills Scientific and Regulatory Affairs, Minneapolis, MN, USA

9 National Food Institute, Technical University of Denmark, Kongens Lyngby, Denmark

10 Jean Mayer USDA Human Nutrition Research Center on Aging at Tufts University, Boston, MA, USA

11 Friedman School of Nutrition Science and Policy, Tufts University, Boston, MA, USA 


$\begin{array}{ll}\text { FSA } & \text { Food Standards Agency } \\ \text { FVNLO } & \text { Fruit, vegetables, nuts, legumes, oils } \\ \text { HDS } & \text { Healthy Diet Score } \\ \text { HEI } & \text { Healthy Eating Index } \\ \text { HEIFA } & \text { Healthy Eating Index for Australian Adults } \\ \text { INCA } & \begin{array}{l}\text { Etude Individuelle Nationale des Consomma- } \\ \text { tions Alimentaires }\end{array} \\ \text { NDNS } & \text { National Diet and Nutrition Survey } \\ \text { NHANES } & \text { National Health and Nutrition Examination } \\ & \text { Survey } \\ \text { NNPAS } & \text { National Nutrition and Physical Activity } \\ & \text { Survey } \\ \text { NP } & \text { Nutrient profiling } \\ P N N S-G S & \text { Programme National Nutrition Santé Guide- } \\ & \text { line Score } \\ U K & \text { United Kingdom } \\ U S & \text { United States } \\ W G I & \text { Whole Grain Initiative }\end{array}$

\section{Introduction}

Poor quality diets are associated with a higher risk of noncommunicable diseases and mortality [1]. The Global Burden of Disease study estimated that diets low in whole grains and fruit and high in sodium accounted for more than half of diet-related deaths in 2017, and that the lack of whole grains in the diet may be the primary dietary contributor to cardiovascular mortality and disability-adjusted life years (DALYs) [1]. In an effort to promote positive dietary habits, dietary guidelines across the globe provide advice on consuming more whole grains, fruit, vegetables, and legumes and limiting sodium, added sugars, and saturated fat [2]. The health benefits of whole grains may be attributed in part to cereal fibre, yet other whole-grain components, such as magnesium and polyphenols, may deliver potential health benefits [3, 4]. Dietary guidelines have encouraged the intake of whole grains, either as quantified recommendations $[5,6]$, or generically linked to choices within a food group [7-9]. Despite well-documented benefits and recommendations, whole-grain consumption remains low in most countries [10-15].

Nutrient profiling (NP) systems can be used to assist consumers in identifying healthier food choices. Ideally, NP systems used for front-of-pack (FOP) labelling assist consumers to make better food choices and promote adherence to dietary guidelines [16]. Most NP systems used for nutrition policy and regulation tend to focus on limiting the content of energy, saturated fat, sugar, and sodium in foods, with some systems promoting beneficial nutrients and foods such as fibre, fruit, and vegetables. However, it is clear there are gaps in the alignment of NP systems with dietary guidelines, particularly for incorporating whole grains in the diet [17].
In principle, we believe only an NP system that accounts for healthful food items including whole grain, while discouraging deleterious components will best support healthy dietary patterns.

Despite the abundance of dietary recommendations, whole grains are not routinely included in NP systems. Systems such as the Health Star Rating (Australia) and Nutri-Score (France, Belgium, Germany, Luxembourg, The Netherlands, Spain, and Switzerland) are based on the Food Standards Agency (FSA)/Ofcom model (United Kingdom (UK)). These systems are designed to encourage the intake of 'beneficial' components such as protein, fibre, fruit, vegetables, nuts, and legumes, while discouraging the intake of 'detrimental' components such as energy, saturated fat, sugar, and sodium. The whole-grain content of foods is currently not included in algorithms for these models. The Nordic Keyhole label is the only FOP NP system to include whole grain within its algorithm. Foods displaying the Keyhole label must fulfil certain conditions around the content of whole grain in addition to fat, sugar, salt, dietary fibre, fruit, and vegetables [18].

It is postulated that including whole grains in NP systems would substantially contribute to an improvement in whole-diet quality and hold relevance in encouraging and promoting greater whole-grain intake at the population level [17]. The aim of this study was to apply modifications to the Nutri-Score algorithm to include whole grain as a beneficial dietary component and to determine whether the Nutri-Score algorithm once modified would improve a theoretical measurement of food and diet quality.

\section{Methods}

Briefly, this study involved development and preliminary analysis of modifications to the Nutri-Score algorithm to include the whole-grain content of foods as an additional beneficial component in the algorithm. We selected the Nutri-Score NP system due to current use in the European Union, with the potential to become the mandatory FOP nutrition labelling system. The original Nutri-Score algorithm and modifications were applied to food composition and dietary intake data from Australia, France, the UK, and the US as examples.

\section{Food composition data}

Food composition data were derived from the Australian Food, Supplement and Nutrient Database (AUSNUT) 2011-2013 [19], the French CIQUAL 2013 food composition database [20], the UK National Diet and Nutrition Survey (NDNS) Nutrient Databank (years 1-6) [21], the NHANES 2015-16 Day 1 Individual Foods dataset [22], 
and the USDA Food Patterns Equivalents Database (FPED) 2015-2016 [23].

In the Australian context, whole-grain content of foods was obtained from the Australian whole-grain database [12, 24]. Based on AUSNUT 2011-2013, which contains 5740 food items, this database utilises a unique coding system to match foods consumed in the Australian National Nutrition and Physical Activity Survey (NNPAS) 2011-2012. For French data, the whole-grain content of foods was estimated from the Etude Individuelle Nationale des Consommations Alimentaires 2 (INCA2) recipe database [25] which provides a list and quantities of ingredients for each composite food. Each whole-grain ingredient was identified to estimate the whole-grain content of foods. For analysis of UK data, the whole-grain content of foods was obtained from the UK whole-grain database [4], based on the UK NDNS Nutrient Databank of approximately 5900 foods. For US data, whole-grain content values were obtained by converting the ounce equivalents from the FPED 2015-2016 to grams. This database converts the foods and beverages contained within the Food and Nutrient Database for Dietary Studies (FNDDS) [26] to 37 food groups and subgroups, including whole grains.

\section{Application of the original Nutri-Score algorithm}

The Nutri-Score NP system is a five-colour FOP nutrition label derived from the UK FSA nutrient profiling system (FSA-NPS) with the nutritional score ranging from - 15 (better nutritional score) to +40 (poorer nutritional score) [27]. For each food or beverage item, the Nutri-Score algorithm allocates up to +10 points individually for energy $(\mathrm{kJ})$, saturated fatty acids (g), total sugar (g), and sodium (mg); while allocating up to -5 points individually for protein $(\mathrm{g})$ and fibre (g), and for fruit, vegetables, nuts, legumes, and walnut/rapeseed/olive oils combined (g) (FVNLO). The point-scoring systems for each individual component vary depending on classification of the food as 'solid food', 'beverage', 'cheese', or 'added fat' [28]. The overall Nutri-Score value is the sum of the scores from individual components, which is then divided into five classes of nutritional quality ranging from A (green-most healthy, lowest nutritional score) to E (red-least healthy, highest nutritional score). Although Nutri-Score is intended for individual foods, the current study calculated Nutri-Score for both individual foods (hereafter referred to as 'food nutritional score') and as a whole-diet nutritional score of individuals using previously validated methods [29].

The original Nutri-Score algorithm was applied to all foods and beverages within the food composition databases described above. The following food items were excluded: alcoholic beverages, beverages/powders for medical purposes, supplements, medicines, caffeine, and single vitamin/ mineral/nutrient items. The Nutri-Score Frequently Asked Questions (FAQ) document was strictly followed to classify and estimate the Nutri-Score of all products [28]. Assumptions made for country-specific databases are described in Supplemental Table 1. Specifically, the Australian Health Survey-Australian Dietary Guidelines database [30], Australian nut database [31], and the AUSNUT 2011-2013 food recipe file [32] were utilised to calculate the FVNLO content of Australian foods. In the UK context, the FSA Standard Recipes Database 1992-2012 [33] was used in conjunction with the NDNS Nutrient Databank to calculate the FVNLO content of foods. For France, the INCA2 recipe database was used to estimate the amount of FVNLO in complex dishes and nutrients of foods were derived from CIQUAL 2013. For the US, the NHANES 2015-2016 Day 1 Individual Foods dataset, the corresponding 2015-2016 FNDDS ingredients dataset, and the FPED 2015-2016 were utilised to estimate FVNLO content of the foods reported consumed by individuals in the NHANES 2015-2016.

\section{Application of modifications to the original Nutri-Score algorithm}

We considered the most recent recommendations for a whole-grain food definition from the Whole Grain Initiative (WGI) in application of the whole-grain modification to the Nutri-Score algorithm [34]. This definition, endorsed by the International Association for Cereal Science and Technology, the Healthgrain Forum, and Cereals and Grains Association, states that to be considered a whole-grain food, a food item shall contain at least $50 \%$ whole-grain ingredients based on dry weight. Furthermore, it is suggested that only foods containing a minimum of $25 \%$ whole-grain ingredients based on dry weight, may make an FOP claim on the presence of whole grain, but cannot be designated "whole grain' in the product name. For inclusion of whole grain into the Nutri-Score algorithm, we applied the following steps. (1) The whole-grain percentage cut-offs outlined by the WGI were included in the beneficial points component (similar to other health-promoting nutrients and foods, e.g., fibre, fruits, and vegetables) of the Nutri-Score algorithm. Up to -5 points were allocated using a non-linear sliding scale, to all foods containing $25-100 \%$ whole grain on a dryweight basis (Table 1). The higher negative score allocated for $\geq 50 \%$ was used as a possible mechanism to incentivise food manufacturers to meet the 'whole-grain food' target. (2) Whole-grain content on a dry-weight basis was calculated for foods within each country-specific food composition database. Dry-weight calculations are published elsewhere [12]. (3) The whole-grain points' component was only applied to food items classified as 'solid food'. The modified Nutri-Score algorithm, like the original, was applied to the same food composition data outlined above. 
Table 1 Summary of the wholegrain modification to NutriScore algorithm

\begin{tabular}{lllllll}
\hline Score & 0 & -1 & -2 & -3 & -4 & -5 \\
\hline Whole-grain percentage (dry weight) $^{\mathrm{a}}$ & $<25 \%$ & $\geq 25 \%$ & & $\geq 50 \%$ & & $100 \%$ \\
\hline
\end{tabular}

A lower nutritional score is indicative of better nutritional quality

${ }^{a}$ Whole-grain percentage cut-offs are derived from the Whole Grain Initiative recommendations for a whole-grain food definition

\section{Dietary intake data}

The Australian NNPAS 2011-2012 collected dietary intake data on 12,153 participants aged 2 years and over, through two separate 24 -h recalls. The first 24 -h recall was conducted through face-to-face interviews with computer assistance $(n=12,153)$, while a second 24 -h recall was conducted via telephone $(n=7735)$. Specific details are described elsewhere [35]. Data were analysed from the Australian Health Survey: Nutrition and Physical Activity, 2011-2012 basic confidentialised unit record files dataset. For the purpose of this study, only data from day 1 and adults were used ( $\geq 18$ years, $n=9430$ following exclusions).

For France, data on dietary intakes were obtained from the second French individual cross-sectional food consumption survey (INCA2) utilising a complex sampling design that is described elsewhere $[25,36]$. The INCA2 survey aimed to estimate the amount of food and beverages consumed by individuals in a representative sample of the French population, using a 7-day open-ended food record. Only data from the adult population (18-79 years old, $n=2624$ ) were used.

In the UK, the NDNS rolling programme collected data related to diet and nutrient intake in individuals aged 1.5 years and over, living in private households in the UK through a four-day food diary [37, 38]. A combined 9374 participants from years 1 to 6 (2008-2014) of the survey completed at least 3 of 4 diary days [39, 40]; only data from day 1 and those aged 18 years or over were included in this study ( $n=4946$ following exclusions).

US data were derived from the nationally representative cross-sectional NHANES 2015-2016 which collected dietary information of the non-institutionalised US population [22]. The first 24-h recall was collected in person via a validated automated multiple pass method, while a second 24-h recall was conducted via telephone. Only data recorded by adults ( $\geq 18$ years; $n=5266$ after exclusions) from the first 24-h recall were included in this study, consistent with a single day of data from the Australian and UK analyses.

\section{Estimation of whole-diet nutritional scores}

The original and modified Nutri-Score algorithm was applied to national dietary intake data to determine the whole-diet nutritional score for individuals. This was calculated based on the sum of individual nutritional scores of foods by consumed energy density over total energy consumed (Calculation 1) [29]. Water and other foods/beverages with zero energy content were excluded from analyses and participant data that reported consumption of these foods/ beverages only were excluded from analyses.

\section{Calculation 1}

Whole-diet nutritional score $=\frac{\sum_{i=1}^{n} \mathrm{FS}_{i} E_{i}}{\sum_{i=1}^{n} E_{i}}$.

$\mathrm{FS}_{i}$ represents the food or beverage nutritional score, and $E_{i}$ represents energy intake from the food or beverage. An increase in whole-diet nutritional score reflects decreasing quality from foods consumed.

\section{Statistical analysis}

Statistical analyses were performed using Stata (StataCorp Stata Statistical Software: Release 15, 2017, College Station, TX, USA) and SAS (SAS Institute Inc: Version 9.4, Cary, NC, USA).

We compared values derived through the original and modified Nutri-Score algorithms, specifically investigating the correlation between individual components of the score (total energy, saturated fat, sugar, sodium, whole grain, fibre, protein, and FVNLO) and the final food nutritional score through Spearman's correlations. Additionally, the changes to food nutritional score and Nutri-Score classes of all foods and those within grain-specific food groups were determined.

To identify the extent to which the original and modified Nutri-Score algorithms aligned with diet-quality scores, population-specific diet-quality scores were compared with whole-diet nutritional scores of individuals through Pearson's correlations. These included the Healthy Eating Index for Australian Adults (HEIFA) [41], the simplified Programme National Nutrition Santé Guideline Score (PNNSGS) 2 [42], the modified Healthy Diet Score (HDS) [43], and the US Healthy Eating Index (HEI) 2015 [44] for Australia, France, the UK, and the US, respectively. These diet-quality scores are based on dietary intake guidelines and recommendations within each country, where a higher score is reflective of better diet quality. For the modified HDS, we 
Fig. 1 Effect of whole-grain modification to Nutri-Score class for all foods compared with the original Nutri-Score algorithm for Australia (A; $n=5647)$, France $(\mathbf{B} ; n=1304)$, the UK $(\mathbf{C} ; n=5261)$, and the US $(\mathbf{D} ; n=5011)^{\mathrm{ab}}$. ${ }^{\mathrm{a}}$ Higher Nutri-Score class denotes better nutritional quality of foods. ${ }^{\mathrm{b}}$ France has a different scale for $\mathrm{y}$-axis due to a minimal number of included foods

used Englyst fibre values based on previous dietary fibre recommendations in the UK $[45,46]$. Correlations were considered significant if $P<0.05$.

Data were converted into non-consumers and quartiles of whole-grain intake (per $10 \mathrm{MJ}$ of energy intake/day) to determine the association between whole-grain intake and whole-diet nutritional score for the original and modified algorithms. Quantile analyses were performed using ANOVA with overall significance determined at $P<0.05$. Mean diet-quality scores for whole-grain intake groups were calculated and compared against the associations between whole-grain intake and whole-diet nutritional score.

\section{Results}

\section{Application of the original Nutri-Score algorithm}

Following exclusions, 5647, 1304, 5261, and 5011 foods from Australian, French, UK and US food composition datasets were included in the country-specific analyses. Application of the original Nutri-Score algorithm exhibited similar distributions of Nutri-Score class A to E across all datasets with the highest number of foods scoring a NutriScore A (Fig. 1). When including all foods, correlations between content of components in the food (in $\mathrm{g}$, $\mathrm{mg}$, or $\mathrm{kJ}$ ) and total nutritional score of the food indicate detrimental components, particularly energy and saturated fat, were the predominant drivers of food nutritional score (Table 2). However, there were some differences between food types (Supplemental Table 2). For example, foods in the composite 'cereal and cereal products' food group in Australian data, which included bread, pasta, breakfast cereal, and individual grain food products, had a correlation of 0.26 between energy content and food nutritional score, while for the 'cereal based products and dishes' sub-category, including biscuits, cakes, sandwiches, and other mixed dishes, the correlation was 0.87 . Food groups containing both wet and dry cereal varieties, such as the 'cereal and cereal products' food group, tended to have a lower correlation between energy content and overall food nutritional score likely due to the large variation in energy density of foods within this food group [17]. Interestingly, positive correlations for components that would result in a better nutritional quality score, including fibre, protein, and FVNLO, were found across datasets (Table 2, Supplemental Table 2).
A

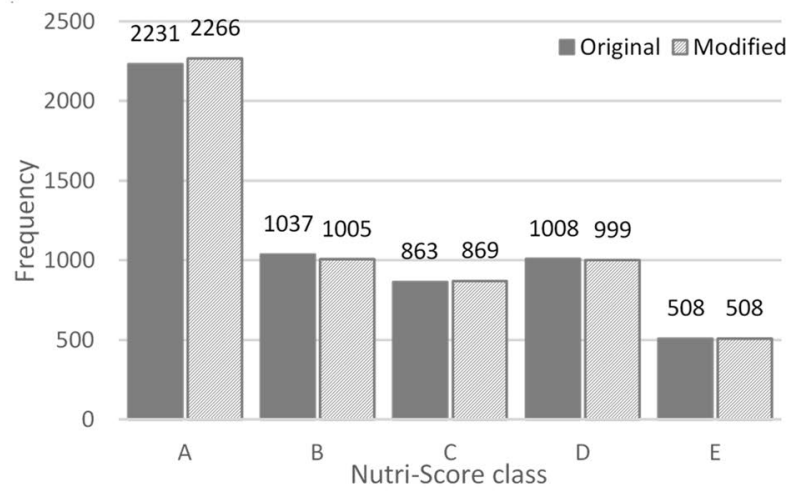

B

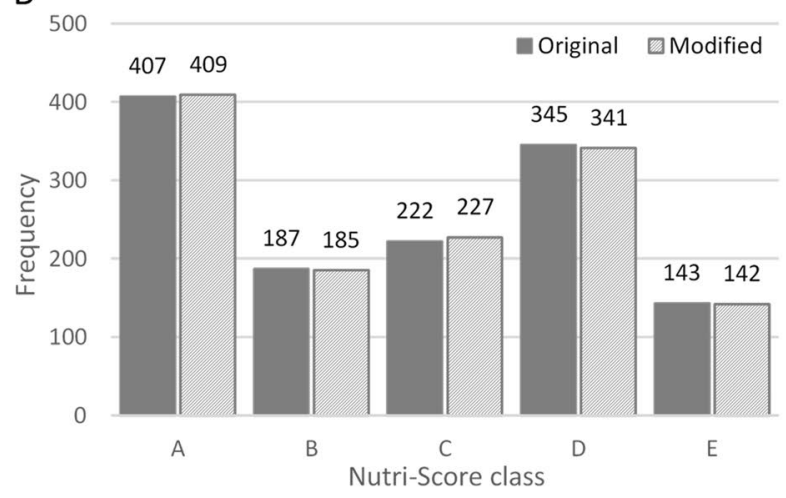

C
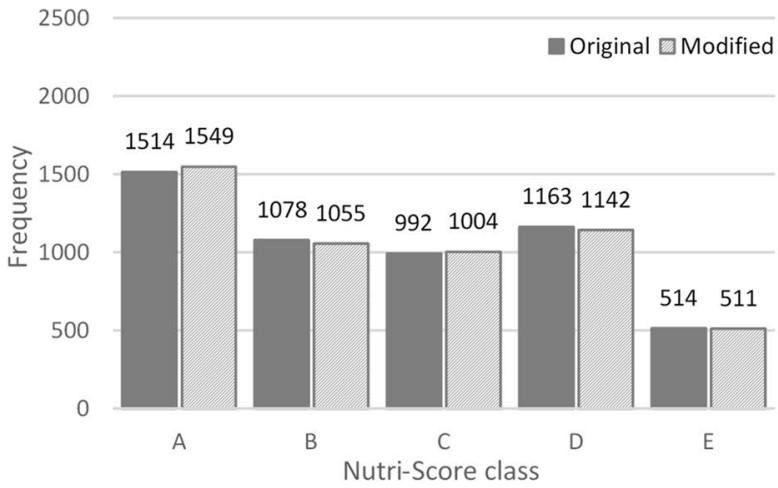

D
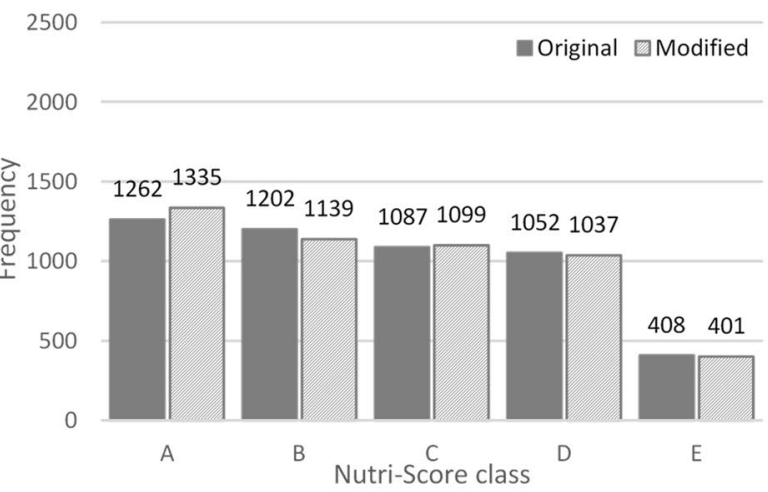


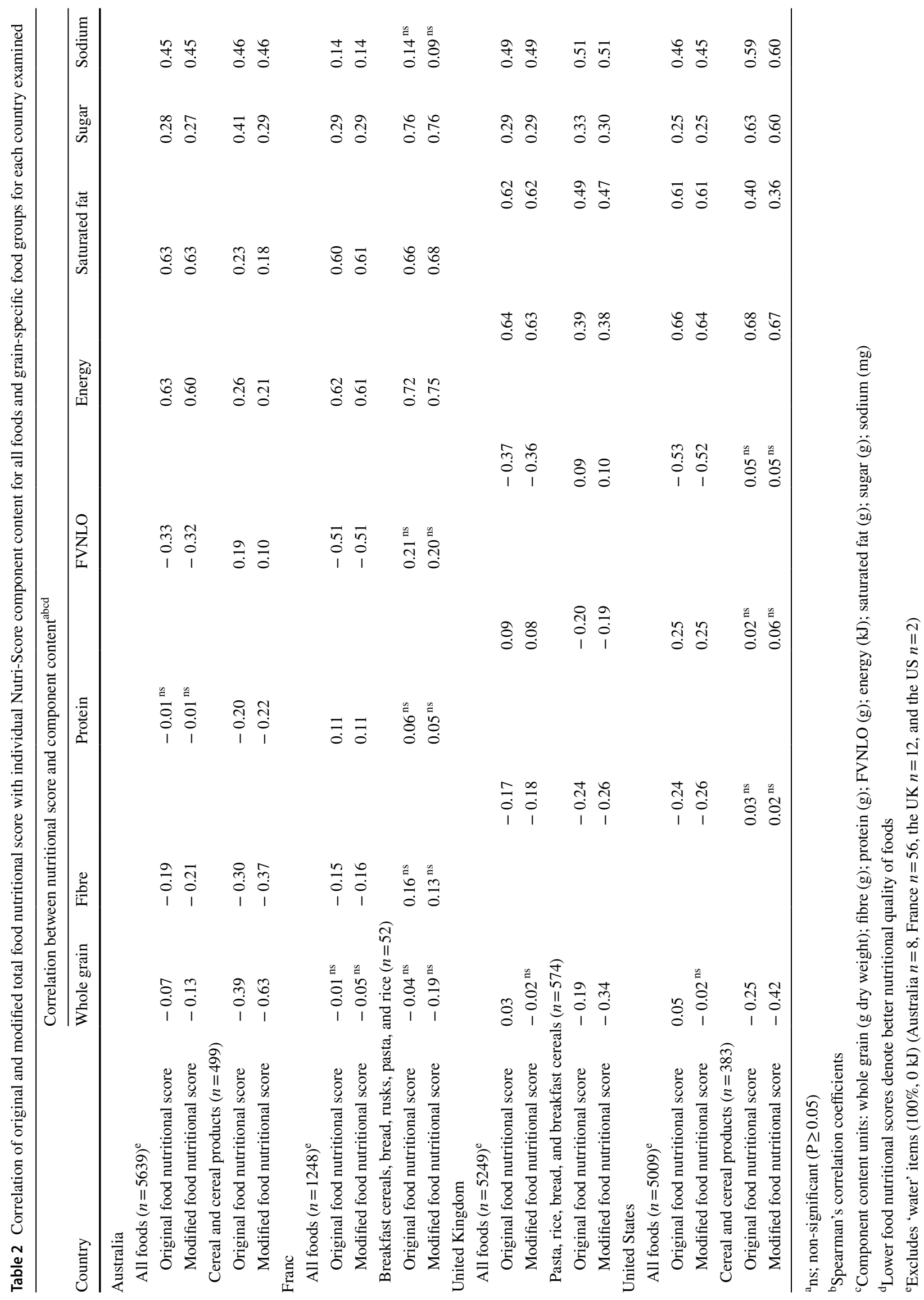


Fig. 2 Effect of whole-grain modification to Nutri-Score class for grain-specific food groups compared with the original Nutri-Score algorithm for Australia (A; $n=499)$, France $(\mathbf{B} ; n=52)$, the UK (C; $n=574)$, and the US $(\mathbf{D} ; n=383)^{\text {ab }}{ }^{\text {a Higher Nutri-Score class }}$ denotes better nutritional quality of foods. ${ }^{\mathrm{b}}$ France has a different scale for $y$-axis due to a minimal number of included foods

Prior to inclusion of whole-grain content "points" in the modified algorithm, and for all foods, whole-grain content was inconsistently correlated with the original food nutritional score across the datasets (Table 2). Generally, for grain-specific food groups, there were more robust negative correlations; that is, higher whole-grain content was correlated with an improved food nutritional score leading to a higher, more healthful Nutri-Score class (Australia - 0.39, US - 0.25, UK - 0.19; Table 2). However, there was no correlation in the French data where a low number of wholegrain foods were included.

\section{Application of the modified Nutri-Score algorithm}

The addition of whole grain to the Nutri-Score algorithm made little difference to the correlations between the content of individual scored components (fibre, energy, saturated fat, etc.) and food nutritional score when considering all foods (Table 2). That is, including the content of whole grain from grain-based foods is not substantial enough to change the overall nutritional score for all foods. In addition, the correlation for whole-grain content and modified Nutri-Score within all foods was weakly negative across all countries; less than -0.10 except for Australia ( -0.19$)$. However, as expected, the correlation for whole-grain content as a contributor to better nutritional quality of foods was strengthened in grain-specific food groups with the modified Nutri-Score algorithm (Table 2, Supplemental Table 2). This was consistent across all datasets, with improvements in correlation ranging from $61.5 \%$ to $375 \%$ improvement (difference in correlation Australia - 0.24, 61.5\%; France - 0.15, $375 \%$; UK - 0.15, 78.9\%; US - 0.17, 68.0\%).

Application of the modified Nutri-Score algorithm resulted in minimal change in Nutri-Score class across all foods (Fig. 1). However, this shift was magnified when investigating some grain-specific food groups, namely those with a higher frequency of foods containing whole grain (Fig. 2). For grain-specific food groups, the wholegrain modification predominately shifted foods from NutriScore class B to A, while those in Nutri-Score class C, D, and $\mathrm{E}$ showed limited change. For example, in Australia, $6.4 \%$ of foods in the 'cereal and cereal products' food group changed from Nutri-Score class B to A, while $0.8 \%$ changed from $\mathrm{D}$ to $\mathrm{C}$ and $0.6 \%$ from $\mathrm{C}$ to $\mathrm{B}$. Foods which changed from $\mathrm{B}$ to $\mathrm{A}$ were predominately whole meal or mixed grain breads, flavoured porridge, and breakfast cereals or muesli

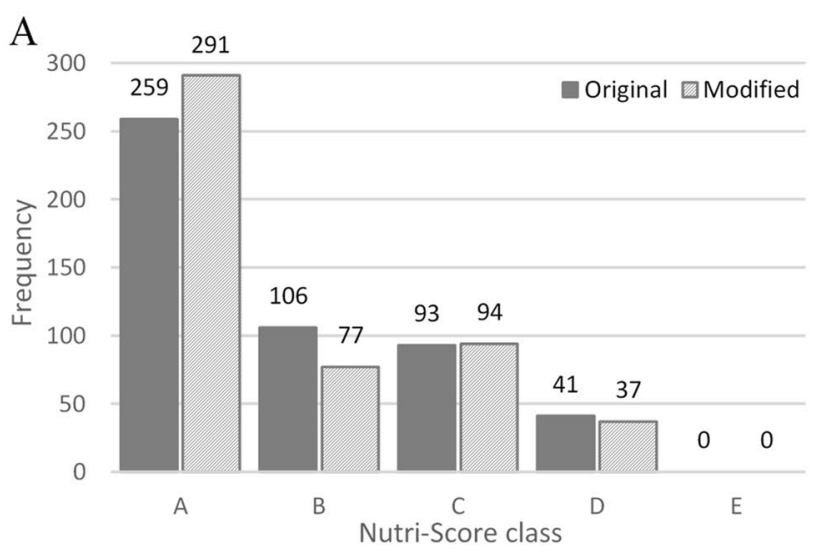

B
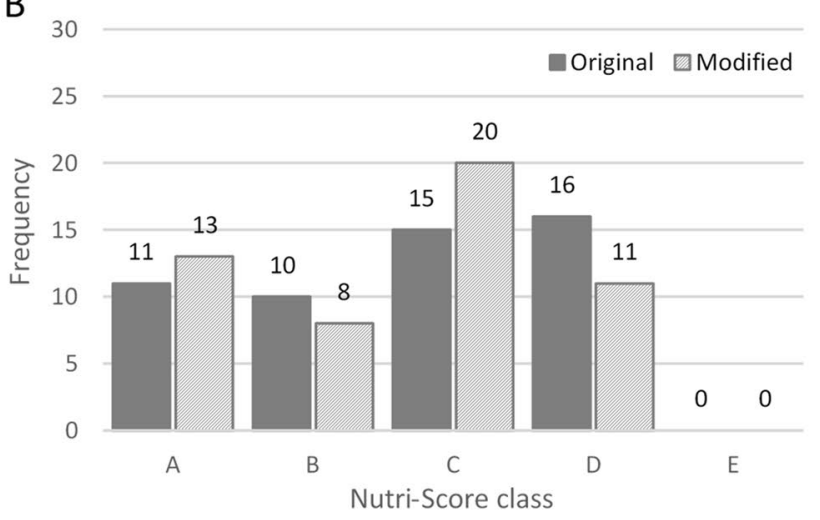

C

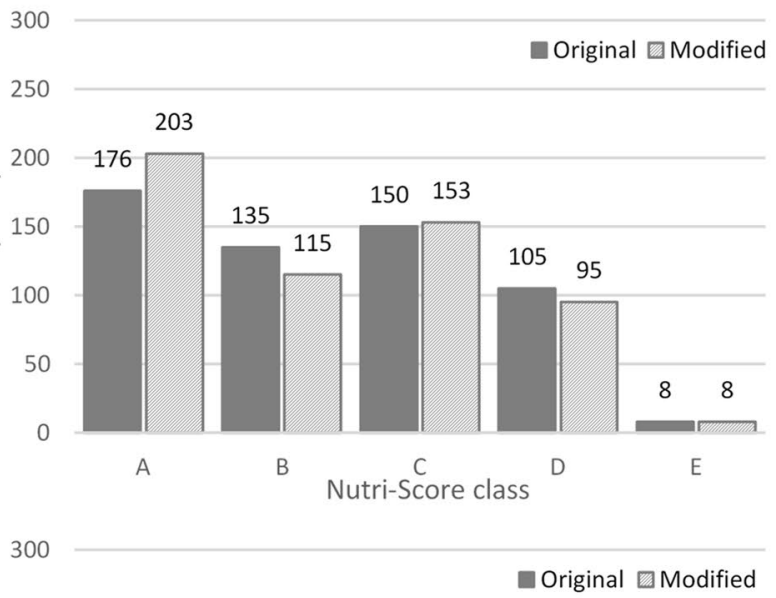

250

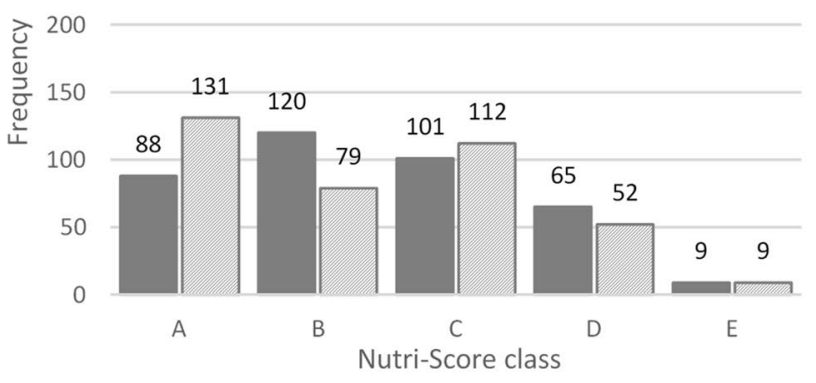


with dried fruit. Based on the Nutri-Score individual component points (out of 10), 94\% of these foods scored $\leq 5$ points $(\leq 27 \mathrm{~g} / 100 \mathrm{~g})$ for sugar with $50 \%$ scoring between 0 and 2 points $(0-13.5 \mathrm{~g} / 100 \mathrm{~g}) ; 100 \%$ scored $\leq 2$ points $(\leq 3 \mathrm{~g} / 100 \mathrm{~g})$ for saturated fat; and $88 \%$ scored $\leq 5$ points $(\leq 540 \mathrm{mg} / 100 \mathrm{~g})$ for sodium with $66 \%$ scoring between $0-1$ points $(0-180 \mathrm{mg} / 100 \mathrm{~g})$.

While a high percentage of grain-specific food groups containing breakfast cereals, bread, rice, and pasta had a shift in food nutritional score (for example, $36.4 \%$ in UK data to $62.5 \%$ in French data), the shift in score did not change Nutri-Score class to the same extent (the UK 7.7\%; Australia 7.8\%; the US 15.1\%; and France 20.8\%) (Table 3).

\section{Alignment of original and modified Nutri-Score algorithms with country-specific diet-quality scores}

The correlations between whole-diet nutritional score and country-specific diet-quality scores improved across all datasets with the whole-grain modification (Table 4). The change in correlation was small with only a maximum of 0.02 improvement.
In general, there was an inverse linear trend showing better whole-diet nutritional score values with increasing daily whole-grain intake (Fig. 3). For example, in US data, non-consumers of whole grain had a mean original wholediet nutritional score of 7.88, while the highest quartile of whole-grain intake had a mean original whole-diet nutritional score of 5.32 (lower score indicative of better diet quality) (Supplemental Table 3). With modifications to the Nutri-Score algorithm, whole-diet nutritional scores of whole-grain intake groups were incrementally improved. For example, in UK data, original compared with modified nutritional score across whole-grain consumers were Q1: 7.34 vs 7.31 , Q2: 6.37 vs $6.17, \mathrm{Q} 3: 5.50$ vs 5.17 , Q4: 4.30 vs 3.59. In comparison, there was a positive linear trend for diet-quality scores with increasing whole-grain intake (higher diet-quality score indicative of better diet quality). Similar patterns were seen across country-specific datasets.

Table 3 Effect of whole-grain modification to food nutritional score and Nutri-Score class for all foods and grain-specific food groups compared with original Nutri-Score algorithm

\begin{tabular}{|c|c|c|c|}
\hline & $n^{\mathrm{a}}$ & $\begin{array}{l}\text { Number of foods changing food } \\
\text { nutritional score }\end{array}$ & $\begin{array}{l}\text { Number of foods } \\
\text { changing Nutri-Score } \\
\text { class }^{b}\end{array}$ \\
\hline \multicolumn{4}{|l|}{ Australia } \\
\hline All foods & 5639 & $298(5.3 \%)$ & $47(0.8 \%)$ \\
\hline Cereal and cereal products & 499 & $238(47.7 \%)$ & $39(7.8 \%)$ \\
\hline Cereal based products and dishes & 914 & $26(2.8 \%)$ & $2(0.2 \%)$ \\
\hline Confectionary and cereal/fruit/nut/seed bars & 144 & $14(9.7 \%)$ & $1(0.7 \%)$ \\
\hline \multicolumn{4}{|l|}{ France } \\
\hline All foods & 1304 & $26(2.0 \%)$ & $8(0.6 \%)$ \\
\hline Breakfast cereals & 24 & $15(62.5 \%)$ & $5(20.8 \%)$ \\
\hline Bread, rusks, pasta, and rice & 28 & $9(32.1 \%)$ & $2(7.1 \%)$ \\
\hline Sugared biscuits and cereal bars & 27 & $1(3.7 \%)$ & $1(3.7 \%)$ \\
\hline \multicolumn{4}{|l|}{ United Kingdom } \\
\hline All foods & 5249 & $310(5.9 \%)$ & $75(1.4 \%)$ \\
\hline Pasta, rice, bread, and breakfast cereals & 574 & $209(36.4 \%)$ & $44(7.7 \%)$ \\
\hline Buns, cakes, pastries, fruit pies, and puddings & 442 & $22(5.0 \%)$ & $8(1.8 \%)$ \\
\hline Biscuits and snacks & 186 & $51(27.4 \%)$ & $15(8.1 \%)$ \\
\hline \multicolumn{4}{|l|}{ United States } \\
\hline All foods & 5011 & $394(7.9 \%)$ & $112(2.2 \%)$ \\
\hline Cereal and cereal products & 383 & $196(51.2 \%)$ & $58(15.1 \%)$ \\
\hline Cereal based products and dishes & 622 & $79(12.7 \%)$ & $27(4.3 \%)$ \\
\hline Snack foods/cereal/fruit/bars & 35 & $4(11.4 \%)$ & $1(0 \%)$ \\
\hline
\end{tabular}

Foods containing whole grain are not solely in the grain-specific food groups listed

${ }^{a}$ Excludes 'water' items ( $100 \%, 0 \mathrm{~kJ}$ ) (Australia $n=8$, France $n=56$, the UK $n=12$, and the US $n=2$ )

${ }^{\mathrm{b}}$ Food nutritional score of Nutri-Score class (foods) A: $\leq-1$, B: 0-2, C: 3-10, D: 11-18, E: $\geq 19$; (beverages) A: water, B: $\leq 1$, C: 2-5, D: 6-9, $\mathrm{E}: \geq 10$ 
Table 4 Correlation of wholediet nutritional scores with country-specific diet-quality scores for original and modified Nutri-Score algorithms

\begin{tabular}{|c|c|c|}
\hline & $\begin{array}{l}\text { Mean whole-diet nutritional } \\
\text { score }^{\mathrm{a}}\end{array}$ & $\begin{array}{l}\text { Correlation with country- } \\
\text { specific diet-quality } \\
\text { score }^{\text {b }}\end{array}$ \\
\hline \multicolumn{3}{|l|}{ Australia $(n=9430)$} \\
\hline Original Nutri-Score algorithm & 4.66 & -0.71 \\
\hline Modified Nutri-Score algorithm & 4.38 & -0.72 \\
\hline \multicolumn{3}{|l|}{ France $(n=2624)$} \\
\hline Original Nutri-Score algorithm & 6.62 & -0.40 \\
\hline Modified Nutri-Score algorithm & 6.55 & -0.41 \\
\hline \multicolumn{3}{|l|}{ United Kingdom $(n=4946)^{\mathrm{c}}$} \\
\hline Original Nutri-Score algorithm & 6.09 & -0.37 \\
\hline Modified Nutri-Score algorithm & 5.87 & -0.38 \\
\hline \multicolumn{3}{|l|}{ United States $(n=5266)$} \\
\hline Original Nutri-Score algorithm & 7.42 & -0.60 \\
\hline Modified Nutri-Score algorithm & 7.28 & -0.62 \\
\hline \multicolumn{3}{|c|}{${ }^{a}$ Lower whole-diet nutritional score indicates better nutritional quality of diet } \\
\hline \multicolumn{3}{|c|}{$\begin{array}{l}{ }^{\mathrm{b}} \text { Correlation with Australia: Healthy Eating Index for Australian Adults (HEIFA); France: simplified Pro- } \\
\text { gramme National Nutrition Santé-Guidelines Score } 2 \text { (PNNS-GS2); United Kingdom: modified Healthy } \\
\text { Diet Score (HDS); United States: Healthy Eating Index (HEI) }\end{array}$} \\
\hline \multicolumn{3}{|c|}{$\begin{array}{l}{ }^{c} \text { Modified HDS calculated using Englyst fibre values. Years } 1 \text { to } 6 \text { of UK NDNS Nutrient Databank only } \\
\text { report Englyst fibre values. The range specified in the modified HDS account for previous dietary fibre rec- } \\
\text { ommendations as Englyst values }\end{array}$} \\
\hline
\end{tabular}

\section{Discussion}

This study shows the feasibility of modifying the NutriScore NP algorithm with the addition of whole grain as a beneficial component, to better align with dietary guidelines recommending increased whole-grain intake. The proposed modification led to stronger correlations with national dietquality scores and better differentiation between low and high whole-grain consumers in the four datasets analysed. These improvements, although small, are still relevant in the context of whole-grain promotion to improve intake for global health.

At the food level, the proposed modification strengthened correlations between whole-grain content of foods and more favourable Nutri-Score values. As expected, this was particularly consistent and strengthened among grain-specific food groups.

A potential argument against inclusion of whole grain in $\mathrm{NP}$ algorithms that do not include this food component is that cereal fibre is already captured by dietary fibre scoring in existing systems, and cereal fibre is suggested to be a key attribute to health benefits observed with higher whole-grain intakes. However, observational studies within Australia and the UK have shown associations between whole-grain intake and reductions in cardiovascular disease risk factors even after adjustment for cereal fibre [3, 4]. This suggests that in addition to fibre, other whole-grain components may contribute to beneficial health effects [47]. Here, despite what may be considered double-counting fibre with the addition of whole grain to the Nutri-Score algorithm, stronger correlations were observed for whole grain in most grain-specific food groups, compared with fibre alone. Additionally, the concept of double-counting fibre is already present in NutriScore and other NP systems by including foods containing fibre, such as fruits, vegetables, nuts, and legumes. Moreover, the current maximum fibre threshold in Nutri-Score is low at $4.7 \mathrm{~g} / 100 \mathrm{~g}$ of food, and numerous whole-grain products contain higher fibre levels. For example, $31 \%$ of foods containing whole grain in US data have $>6 \mathrm{~g} / 100 \mathrm{~g}$ of fibre. Therefore, including a whole-grain component may better emphasise the fibre content within whole-grain foods. This notion is consistent with global research showing a diet low in whole grains is attributable to higher rates of mortality and DALYs than a diet low in fibre (3 v 1 million deaths; 82 v 20 million DALYs) [1].

We chose specific cut points for whole-grain scoring to reflect recognised values determined by the WGI as relevant to promote to consumers. That is, foods with a whole-grain content of $<25 \%$ do not receive additional scoring in this algorithm. Ideally, the NP system should only promote foods that are higher in whole grain and in particular those with a very high (50-100\%) whole-grain content. As such, only a minimal number of foods were affected, reflecting the relatively small proportion of high whole-grain foods in these food supplies. In addition, a large majority of foods containing whole grain were awarded Nutri-Score class A prior to modifications to the algorithm, such that improvements to Nutri-Score class could not be detected. 
Fig. 3 Relationship between whole-grain intake, whole-diet nutritional score for the original and modified Nutri-Score algorithm, and national diet-quality score for Australia (A), France (B), the UK (C)

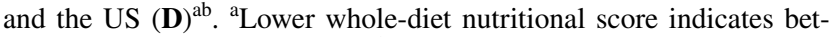
ter nutritional quality of diet. ${ }^{\mathrm{b}} N C$ non-consumers; HEIFA Healthy Eating Index for Australian adults, PNNS-GS2 Programme National Nutrition Santé-Guidelines Score 2, HDS Healthy Diet Score, HEI Healthy Eating Index

Limited improvements to Nutri-Score at the dietary intake level, even with modifications including whole grain, may also be due to the low level of whole-grain consumption in the four countries. For example, foods containing $\geq 25 \%$ whole grain only contribute to $3 \%$ of energy intakes in France, with $56 \%$ of individuals classified as non-consumers of these foods. However, the addition of whole grain to NP algorithms resulting in improved NP scores provides opportunities for manufacturers to promote the benefits of whole grain within products through labelling. This may increase consumer awareness and guide them towards higher and healthier whole-grain choices [48, 49]. It may also incentivise manufacturers to include more whole grain, by replacing refined with whole grain, so that more foods can be called a 'whole-grain food' FOP and benefit from the improved NP score.

Inclusion of whole grain in initiatives such as Nordic Keyhole label has proved beneficial. This NP scheme indicates nutritionally better food choices within food categories. Studies in Sweden and Denmark indicate that replacing non-Keyhole foods with Keyhole equivalents would increase whole-grain intake by $654 \%$ and $76 \%$, respectively [50,51].

At the dietary intake level, the whole-grain modification better reflected improvement of dietary quality and greater alignment with dietary guidelines. Addition of whole grain to NP systems is important to capture all factors contributing to better diet quality, allowing consistency between public health messages on individual foods (Nutri-Score and other $\mathrm{NP}$ ) and broad messages to improve dietary quality such as dietary guidelines.

While the work here suggests that the inclusion of whole grain in NP systems may be beneficial, the implementation of such modifications may prove challenging. First, the methods used to determine whole-grain content within foods varies greatly, including classification of 'whole grain' as an ingredient and the lack of an analytical method to measure whole grain in foods. Some amounts of whole grain may not be included in the datasets used to calculate scores [52, 53]. Second, whole-grain labelling on food products is not consistently regulated, leading to consumer and manufacture confusion [48]. Finally, obtaining whole-grain data for third-party assessment may be difficult as the calculation of ingredient content is not often a regular practice for manufacturers. These problems are common to other components of Nutri-Score including fruit and vegetables. Prior
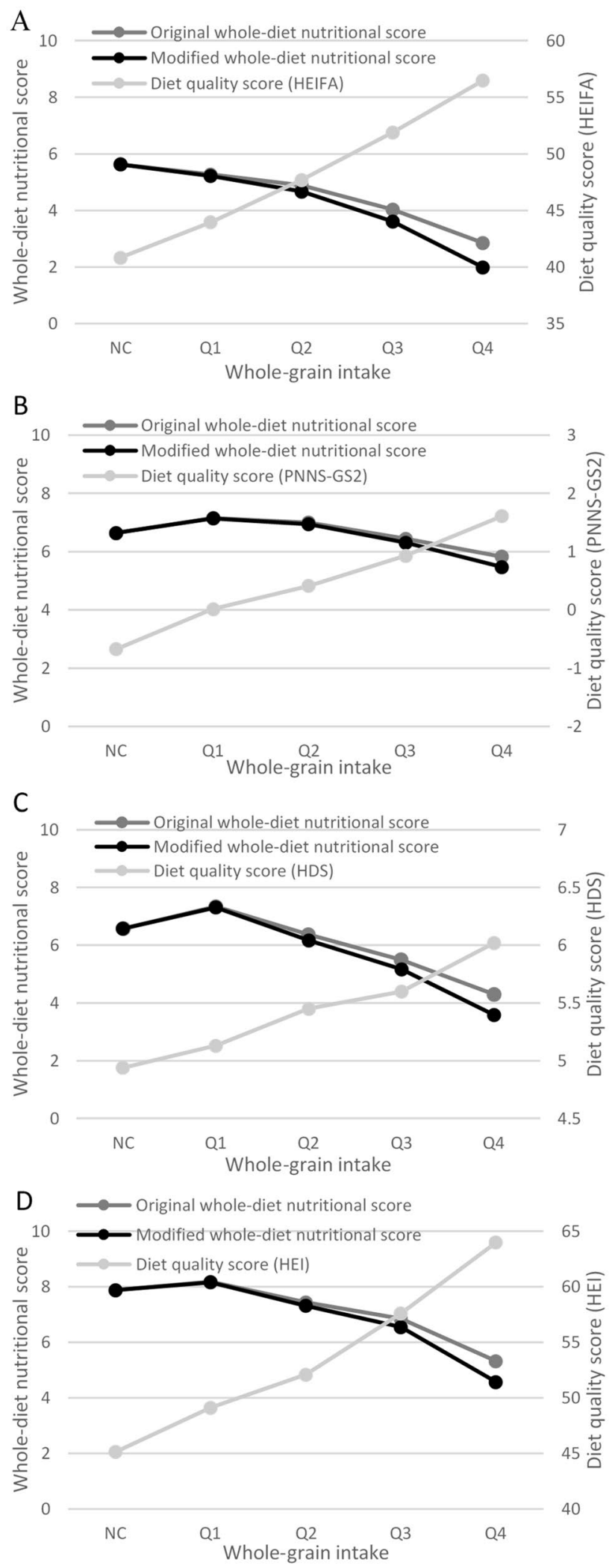

to implementation of modifications, it is important to ensure consistency in the calculation of whole grain within foods, including whole-grain definitions, and to establish consistent 
regulations for whole-grain labelling that NP systems can apply.

Various limitations are present in the current study. In the French food composition database, there were far fewer foods than in other datasets, and likely greater nutritional deviation between foods, potentially leading to larger effect sizes. Similarly, a few foods containing whole grain were included, such that correlations may not be significant. Furthermore, only 1 day of dietary intake data was utilised for Australia, the UK, and the US to maximise the sample size included in analyses. This approach does not provide a reliable estimate of usual intake and as such the interpretation of the results should be based on using estimates of population intake distributions for 'any given day'. For this particular research question, utilising a single day of dietary intake was considered sufficient as this study investigated a comparison of methods over various distributions of intakes across different populations, in which individual dietary intakes were not required to be assessed in detail.

In addition, we did not modify the Nutri-Score algorithm beyond including a whole-grain component. Before implementing the inclusion of whole grain, it would be important to perform a full review of the scoring approach, ensuring that the nutritional score received by foods is balanced within and between food categories. It may be necessary to implement a strategy to penalise foods higher in energy, sugar, sodium, and saturated fat, for example excluding whole-grain points if a specific detrimental point content threshold is met (similar to that for protein). However, analysis of Australian foods in the 'cereal and cereal products' food group changing from Nutri-Score class B to A identified that few foods scored well for whole grain and poorly for sugar and sodium (and none for saturated fat). As such, in any food supply, consideration of penalisation for detrimental components may only be relevant for a few foods, although such scoring is worth investigating.

Moreover, ranges of score used to identify Nutri-Score class were not altered, and foods containing substantial amounts of whole grain may not be well distinguished from those with less whole grain, particularly relevant for class A foods. Previous research found that Nutri-Score could discriminate between whole and refined foods [54], although similar algorithms on the Australian Health Star Rating showed limited potential to make this differentiation [55].

Therefore, future research should consider testing other varied modifications that achieve balance within and between food categories, such as alterations of the scoring approach for individual food groups and nutrient components of the score; changes to the thresholds that determine Nutri-Score class; or inclusion of other nutrients and food groups, including whole grain, that are grounded in dietary guidance. Further optimization of the Nutri-Score may be necessary to adequately achieve its goal of improving population-level diet quality, either through influencing consumer food choice or food product reformulations. In addition, differing country-specific diet-quality scores were included to better reflect the food supply and dietary guidelines of each country. As such, the effects of the modified Nutri-Score algorithm within each context cannot be directly compared. Some diet-quality scores (e.g., HDS) do not consider whole grain in the criteria, meaning that correlations with the modified algorithm may not be as strong.

The addition of whole grain as a beneficial component within the Nutri-Score NP algorithm improves correlations with other dietary quality markers and better differentiates individuals of low and high whole-grain consumption. While improvements to food nutritional score were minimal, improvements are still relevant to better promote whole grain given the extensive evidence on health benefits. Consistency in whole-grain ingredient and food definitions, and regulation on whole-grain labelling are all needed prior to implementation of modifications. A modification to include whole grain in NP algorithms is justified to align with dietary guidelines and better reflect whole grain as a component to measure dietary quality. This study provides a blueprint for further research to test varied modifications to Nutri-Score and other NP systems.

Supplementary Information The online version contains supplementary material available at https://doi.org/10.1007/s00394-021-02718-6.

Author contributions The author's responsibilities were as follows: KRK, FV, KCM, AD, CJS, GM, JS, HM, NMM, and EJB designed research; $\mathrm{KRK}, \mathrm{FV}$, and $\mathrm{KCM}$ conducted research; KRK, FV, and $\mathrm{KCM}$ analysed data; KRK, FV, KCM, AD, CJS, GM, JS, HM, NMM, and EJB wrote the paper; KRK and EJB had primary responsibility for final content. All authors read and approved the final manuscript.

Funding This research was funded by Cereal Partners Worldwide and General Mills, Inc.

\section{Declarations}

Conflict of interest FV is employed by MS-Nutrition. AD has received grants, contracts, and honoraria from entities, both public and private, with an interest in nutrient density of foods, meals, and the total diet. GM is employed by Cereal Partners Worldwide. JS is employed by General Mills, Inc. NMM has received funding for investigator-initiated grants from General Mills Bell Institute of Health and Nutrition, received speaker honoraria from Cereal Partners Worldwide and General Mills, Inc., is a member of the working group of the Whole Grain Initiative, and serves as an unpaid scientific advisor on the Oldways Whole Grains Council. EJB has received funding for presentations on whole grain by Quaker Oats and General Mills, Inc., and research funding from Cereals Partners Worldwide. She is a member of the working group of the Whole Grain Initiative. CJS is retired and has no conflicts of interest to report; previously, he has received funding for investigator-initiated grants from Cereal Partners Worldwide and Kel$\operatorname{logg}$ 's, manufacturers of whole-grain breakfast cereals. He is a member of the European Healthgrain Forum and the Definitions Working 
Group of the Whole Grain Initiative. All the other authors report no conflicts of interest.

Availability of data and materials Data described in the manuscript will be made available (where possible) upon request. Raw data relating to the Australian National Nutrition and Physical Activity Survey 2011-2012 and all UK data require access approval by the Australian Bureau of Statistics and UK Data Service, respectively.

Code availability Code used in the statistical analysis will be made available (where possible) upon request.

Ethics approval All surveys obtained full ethical approval from the necessary committees and comply with the guidelines from the Declaration of Helsinki. Permission to use and analyse the data was obtained through approved registration at the Australian Bureau of Statistics registration centre (Australia) and the UK Data Service (the UK). French and US data are publicly available.

Open Access This article is licensed under a Creative Commons Attribution 4.0 International License, which permits use, sharing, adaptation, distribution and reproduction in any medium or format, as long as you give appropriate credit to the original author(s) and the source, provide a link to the Creative Commons licence, and indicate if changes were made. The images or other third party material in this article are included in the article's Creative Commons licence, unless indicated otherwise in a credit line to the material. If material is not included in the article's Creative Commons licence and your intended use is not permitted by statutory regulation or exceeds the permitted use, you will need to obtain permission directly from the copyright holder. To view a copy of this licence, visit http://creativecommons.org/licenses/by/4.0/.

\section{References}

1. GBD 2017 Diet Collaborators (2019) Health effects of dietary risks in 195 countries, 1990-2017: a systematic analysis for the Global Burden of Disease Study 2017. Lancet 393:1958-1972. https://doi.org/10.1016/S0140-6736(19)30041-8

2. Food and Agriculture Organization of the United Nations (FAO) (2020) Food-based dietary guidelines. http://www.fao.org/nutri tion/education/food-dietary-guidelines/en/. Accessed Oct 2020.

3. Barrett E, Batterham M, Beck E (2020) Whole grain and cereal fibre intake in the Australian Health Survey: associations to CVD risk factors. Public Health Nutr 23(8):1404-1413. https://doi.org/ 10.1017/S1368980019004233

4. Barrett E, Amoutzopoulos B, Batterham M, Ray S, Beck E (2020) Whole grain intake compared with cereal fibre intake in association to CVD risk factors: a cross-sectional analysis of the National Diet and Nutrition Survey (UK). Public Health Nutr 23(8):13921403. https://doi.org/10.1017/S1368980019004221

5. Ministry of Food, Agriculture and Fisheries: Danish Veterinary and Food Administration (2020) The official dietary guidelinesGood for health and climate. Glostrup, Denmark.

6. Superior Health Council (2019) Dietary guidelines for the Belgian adult population. Belgium, Brussels

7. National Health and Medical Research Council (NHMRC) (2013) Eat for health: Australian dietary guidelines. Canberra, Australia.

8. Ministry of Social Affairs and Health. Programme National Nutrition Santé 2019-2023 [French Nutrition and Health Program]. Paris, France

9. Public Health England (2016) The eatwell guide

10. Micha R, Khatibzadeh S, Shi P, Andrews K, Engell R, Mozaffarian D (2015) Global, regional and national consumption of major food groups in 1990 and 2010: a systematic analysis including 266 country-specific nutrition surveys worldwide. BMJ Open 5(9):e008705. https://doi.org/10.1136/bmjopen-2015-008705

11. Miller K (2020) Review of whole grain and dietary fiber recommendations and intake levels in different countries. Nutr Rev 78(S1):29-36. https://doi.org/10.1093/nutrit/nuz052

12. Kissock KR, Neale EP, Beck EJ (2020) The relevance of whole grain food definitions in estimation of whole grain intake: a secondary analysis of the National Nutrition and Physical Activity Survey 2011-2012. Public Health Nutr 23(8):1307-1319. https:// doi.org/10.1017/S1368980019004452

13. Mann KD, Pearce MS, McKevith B, Thielecke F (2015) Low whole grain intake in the UK: results from the National Diet and Nutrition Survey rolling programme 2008-11. Br J Nutr 113(10):1643-1651. https://doi.org/10.1017/S00071145150004 22

14. Albertson A, Reicks M, Joshi N, Gugger C (2016) Whole grain consumption trends and associations with body weight measures in the United States: results from the cross sectional National Health and Nutrition Examination Survey 2001-2012. Nutr J. https://doi.org/10.1186/s12937-016-0126-4

15. Bellisle F, Hebel P, Colin J, Reye B (2014) Consumption of whole grains in French children, adolescents and adults. Br J Nutr 112(10):1674-1684. https://doi.org/10.1186/s12937-016-0126-4

16. Gerrior S (2010) Nutrient profiling systems: are science and the consumer connected? Am J Clin Nutr 91(4):1116-1117. https:// doi.org/10.3945/ajen.2010.28450G

17. Drewnowski A, McKeown N, Kissock K, Beck E, Mejborn H, Vieux F, Smith J, Masset G, Seal C (2021) Why whole grains should be incorporated into nutrient profile models to better capture nutrient density. Adv Nutr 12(3):600-608. https://doi.org/10. 1093/advances/nmaa172

18. Swedish Food Agency (2015) The National Food Agency's Code of Statutes: Regulations amending the National Food Agency's regulations (SLVFS 2005:9) on the use of a particular symbol.

19. Food Standards Australia New Zealand (FSANZ) (2014) AUSNUT 2011-2013

20. The French Agency for Food, Environmental and occupational Health and Safety (ANSES) (2013) French food composition table (CIQUAL)

21. NatCen Social Research, Elsie Widdowson Laboratory MRC (2019) National diet and nutrition survey years 1-9 2008/09-2016/17. UK Data Service. https://doi.org/10.5255/ UKDA-SN-6533-15

22. Centers for Disease Control and Prevention (CDC), National Center for Health Statistics (NCHS). National Health and Nutrition Examination Survey (NHANES) 2015-2016. https://wwwn. cdc.gov/nchs/nhanes/continuousnhanes/default.aspx?BeginYear= 2015. Accessed Oct 2020

23. U.S. Department of Agriculture, Agricultural Research Service, Beltsville Human Nutrition Research Center, Food Surveys Research Group (2018) Food patterns equivalent database 2015-2016. http://www.ars.usda.gov/nea/bhnrc/fsrg. Accessed Oct 2020.

24. Galea LM, Dalton SMC, Beck EJ, Cashman CJ, Probst YC (2016) Update of a database for estimation of whole grain content of foods in Australia. J Food Compost Anal 50:23-29. https://doi. org/10.1017/S1368980017001082

25. French Food Safety Agency (AFSSA) (2009) Etude Individuelle Nationale des Consommations Alimentaires 2 (INCA2) 2006-2007 [Individual National Study of Food Consumption 2006-2007].

26. U.S. Department of Agriculture, Agricultural Research Service (2018) USDA Food and Nutrient Database for Dietary Studies 2015-2016. http://www.ars.usda.gov/nea/bhnrc/fsrg. Accessed Oct 2020 
27. Julia C, Hercberg S (2017) Development of a new front-of-pack nutrition label in France: the five-colour Nutri-Score. Public Health Panor 3(4):537-820

28. Santé Publique France (2020) Nutri-Score frequently asked questions-Scientific \& Technical

29. Julia C, Touvier M, Mejean C, Ducrot P, Peneau S, Hercberg S, Kesse-Guyot E (2014) Development and validation of an individual dietary index based on the British food standard agency nutrient profiling system in a French context. J Nutr 144(12):20092017. https://doi.org/10.3945/jn.114.199679

30. Food Standards Australia New Zealand (FSANZ) (2017) Australian Health Survey-Australian Dietary Guidelines database.

31. Nikodijevic C, Probst Y, Neale E (2019) Development of a database for estimation of the nut content of Australian single-ingredient and multi-ingredient foods. J Food Compost Anal. https:// doi.org/10.1016/j.jfca.2019.103236

32. Food Standards Australia New Zealand (FSANZ) (2014) AUSNUT 2011-2013 Food Recipe File.

33. MRC Human Nutrition Research (2017) Food standards agency standard recipes database, 1992-2012. UK Data Service. https:// doi.org/10.5255/UKDA-SN-8159-1

34. Whole Grain Initiative Global Working Group on Whole Grain Definitions (2020) Definition of a whole-grain food. http://www. wholegraininitiative.org/en/1179-wgi-working-groups. Accessed Jan 2021

35. Australian Bureau of Statistics (ABS) (2015) Australian Health Survey: user's guide 2011-2013

36. Dubuisson C, Lioret S, Touvier M, Dufour A, Calamassi-Tran G, Lafay L (2010) Trends in food and nutritional intakes of French adults from 1999 to 2007: results from the INCA surveys. Br J Nutr 103(7):1035-1048. https://doi.org/10.1017/S000711450 9992625

37. NatCen Social Research, MRC Elsie Widdowson Laboratory. National Diet and Nutrition Survey Years 1-4 2008/09-2011/12 User Guide for UK Data (core \& country boost data).

38. NatCen Social Research, MRC Elsie Widdowson Laboratory. National Diet and Nutrition Survey Years 5-6 2012/13-2013/14 User Guide for UK Data.

39. Public Health England (2017) National diet and nutrition survey: results from years 1, 2, 3, 4 (Combined) of the Rolling Programme (2008/09-2011/12).

40. Public Health England (2016) National diet and nutrition survey: results from years 5 and 6 (combined) of the Rolling Programme (2012/2013-2013/2014)

41. Grech A, Sui Z, Siu H, Zheng M, Allman-Farinelli M, Rangan A (2017) Socio-demographic determinants of diet quality in Australian adults using the validated Healthy Eating Index for Australian Adults (HEIFA-2013). Healthcare 5(1):7. https://doi.org/10.3390/ healthcare 5010007

42. Chaltiel D, Adjibade M, Deschamps V, Touvier M, Hercberg S, Julia C, Kesse-Guyot E (2019) Programme National Nutrition Santé - guidelines score 2 (PNNS-GS2): development and validation of a diet quality score reflecting the 2017 French dietary guidelines. Br J Nutr 122(3):331-334. https://doi.org/10.1017/ S0007114519001181
43. Dikariyanto V, Berry S, Francis L, Smith L, Hall W (2021) Whole almond consumption is associated with better diet quality and cardiovascular disease risk factors in the UK adult population: national diet and nutrition survey (NDNS) 2008-2017. Eur J Nutr 60:643-654. https://doi.org/10.1007/s00394-020-02270-9

44. Krebs-Smith S, Pannucci T, Subar A, Kirkpatrick S, Lerman J, Tooze J, Wilson M, Reedy J (2018) Update of the healthy eating index: HEI-2015. J Acad Nutr Diet 118(9):1591-1602. https://doi. org/10.1016/j.jand.2018.05.021

45. Scientific Advisory Committee on Nutrition (2015) Carbohydrates and health. England, London

46. Public Health England (2016) Government dietary recommendations: government recommendations for energy and nutrients for males and females aged 1-18 years and 19+ years. England, London

47. Zhu Y, Sang S (2017) Phytochemicals in whole grain wheat and their health-promoting effects. Mol Nutr Food Res. https://doi.org/ 10.1002/mnfr.201600852

48. Ross AB, van der Kamp J-W, King R, Le K-A, Mejborn H, Seal CJ, Thielecke F (2017) Perspective: a definition for whole-grain food products-recommendations from the Healthgrain Forum. Adv Nutr 8(4):525-531. https://doi.org/10.3945/an.116.014001

49. Ferruzzi MG, Jonnalagadda SS, Liu S, Marquart L, McKeown N, Reicks M, Riccardi G, Seal C, Slavin J, Thielecke F et al (2014) Developing a standard definition of whole-grain foods for dietary recommendations: summary report of a multidisciplinary expert roundtable discussion. Adv Nutr 5(2):164-176. https://doi.org/10. 3945/an.113.005223

50. Amcoff E, Brugård Konde $\AA$, Jansson A, Sanner Färnstrand J (2015) Switch to the Keyhole-it affects nutrient intake. Uppsala, Sweden

51. Biltoft-Jensen A, Ygil K, Kørup K, Christensen T, Fagt S (2015) Potential effect of eating keyhole-labelled products: Focus on intake of nutrients and whole grains. Technical University of Denmark. Søborg, Denmark, National Food Institute

52. Ross A, Kristensen M, Seal C, Jacques P, McKeown N (2015) Recommendations for reporting whole-grain intake in observational and intervention studies. Am J Clin Nutr 101(5):903-907. https://doi.org/10.3945/ajcn.114.098046

53. Kissock KR, Neale EP, Beck EJ (2021) Whole grain food definition effects on determining associations of whole grain intake and body weight changes: a systematic review. Adv Nutr 12(3):693707. https://doi.org/10.1093/advances/nmaa122

54. Dréano-Trécant L, Egnell M, Hercberg S, Galan P, Soudon J, Fialon M, Touvier M, Kesse-Guyot E, Julia C (2020) Performance of the front-of-pack nutrition label Nutri-Score to discriminate the nutritional quality of foods products: a comparative study across 8 European countries. Nutrients 12(5):1303. https://doi.org/10. 3390/nu12051303

55. Curtain F, Grafenauer S (2019) Health star rating in grain foodsdoes it adequately differentiate refined and whole grain foods? Nutrients 11(2):415. https://doi.org/10.3390/nu11020415 